\title{
SPECTRA IN REPRESENTATIONS OF SEMISIMPLE LIE GROUPS
}

\author{
SIDNEY C. SCULL ${ }^{1}$
}

\begin{abstract}
The spectrum of the infinitesimal generator of a one-parameter group of unitary operators arising from a representation of a semisimple Lie group is determined. The support of the spectral measure depends only on whether the group is a group of automorphisms of a bounded symmetric domain.
\end{abstract}

1. Let $\Pi$ be a (strongly) continuous unitary representation of a semisimple Lie group $G$ on a separable Hilbert space $H$. Let $C^{\infty}(\Pi)$ denote the set of all vectors $v \in H$ such that $\Pi(\cdot) v$ is a $C^{\infty}$ function on $G$. Then $C^{\infty}(\Pi)$ is a dense linear subspace of $H$, and contains the dense set of analytic vectors for the representation [6].

We denote the Lie algebra of $G$ by $g_{0}$. If $x \in g_{0},(\Pi(\exp (t x)))$ is a oneparameter group of unitary operators and has a skew-adjoint infinitesimal generator $d \Pi(x)$. Thus $\Pi(\exp (t x))=\exp (t d \Pi(x)), t \in R$. Also $C^{\infty}(\Pi)$ is contained in the domain of $d \Pi(x)$, and is invariant under $d \Pi(x)$ for all $x \in g_{0}$. Moreover, $x \rightarrow d \Pi(x) \mid C^{\infty}(\Pi)$ defines a representation of $g_{0}$ by essentially skew-adjoint operators.

We shall examine the spectrum of the selfadjoint operator $-i d \Pi(x)$ whenever $\exp (t x)$ satisfies a certain noncompactness condition explained below. The information obtained will depend on $G$ but not on $\Pi$ or $x$. More precisely, if $\int \lambda d E_{\lambda}$ is the spectral resolution of $-i d \Pi(x)$, C. C. Moore [5] has shown that the projection valued measure $E$ is absolutely continuous with respect to Lebesgue measure, and its support is either $(-\infty, \infty),(0, \infty)$ or $(-\infty, 0)$. In the first instance, we shall say $-i d \Pi(x)$ has two-sided spectrum, while in the second and third instances we shall say $-i d \Pi(x)$ has one-sided spectrum.

One's feeling, and this is borne out by our investigations, is that most of the time we can expect a two-sided spectrum, and that a one-sided

Received by the editors February 6, 1973.

AMS (MOS) subject classifications (1970). Primary 22D10, 22E45, 47B15; Secondary $32 \mathrm{M} 15,81 \mathrm{~A} 78$.

Key words and phrases. Unitary representation, spectral measure, semisimple Lie group.

1 These results submitted in partial fulfilment of the author's Ph.D. requirements, at the University of California, Berkeley, August 1971.

(c) American Mathematical Society 1973 
spectrum is a rather rare occurrence. Our principal result may be summarized as follows: one-sided spectra occur only when $G$ is a group of automorphisms of a bounded symmetric domain.

This work was prepared under the direction of Calvin Moore, to whom I am deeply indebted. I would also like to thank B. Kostant for making available an outline of his proof of Proposition 3(ii).

2. If $G$ is a semisimple Lie group, its adjoint group $G^{*}$ is the product of a finite number of simple Lie groups $G_{i}^{*}$. Let $p_{i}$ denote the projection of $G$ onto $G_{i}^{*}$. If $H$ is a subgroup of $G$, then following C. C. Moore [5], we say $H$ is totally noncompact if $p_{i}(H)$ has noncompact closure in $G_{i}^{*}$ for each $i$. If $G$ is simple, we shall say, in the above case, that $H$ is noncompact.

In this section and $\S 3$ we shall prove the following:

THEOREM 1. Let $\Pi$ be a continuous irreducible unitary representation of a connected simple Lie group, such that $G$ is not a group of automorphisms of a bounded symmetric domain. Then if $x \in g_{0}$ generates a noncompact one-parameter group $(\exp (t x)),-i d \Pi(x)$ has two-sided spectrum.

We first note that we can reduce questions about the spectrum of $-i d \Pi(x)$ for general elements $x \in g_{0}$ to analogous questions about $\operatorname{ad}(x)$-nilpotent elements.

Proposition 1. If Theorem 1 is valid for every $x \in g_{0}$ such that $\operatorname{ad}(x)$ nilpotent, then it is valid for every $x \in g_{0}$ such that $(\exp (t x))$ is noncompact.

The proof of this proposition is essentially contained in [5].

We now introduce the principle method of demonstrating that adnilpotent elements have two-sided spectra.

Definition. A Lie group $G$ is of type $\mathrm{F}$ if for every ad-nilpotent element $x \in g_{0}$, there exists an element $\Theta \in G$, such that $\operatorname{Ad}(\Theta) x=-x$. In this case we say $\Theta$ flips $x$.

Our crucial proposition is the following

Proposition 2. Let $G$ be a simple Lie group of type $\mathrm{F}$, and let $\Pi$ be a continuous irreducible unitary representation of $G$. Then the spectrum of $-i d \Pi(x)$ is two-sided for every ad-nilpotent element $x \in g_{0}$.

Proof. Suppose on the contrary that the spectrum of $-i d \Pi(x)$ is one-sided, say $(0, \infty)$, for some ad-nilpotent $x \in g_{0}$. Then $\langle\psi,-i d \Pi(x) \psi\rangle \geqq$ 0 for all $\psi \in C^{\infty}(\Pi)$. Now, by assumption, there exists $\Theta \in G$ such that Ad $\Theta(x)=-x$. Replacing $\psi$ above by $\Pi(\Theta)^{-1} \psi$, and using unitarity of $\Pi$, we obtain $\langle\psi, i d \Pi(x) \psi\rangle \geqq 0$ for all $\psi \in C^{\infty}(\Pi)$. This contradicts our original assumption about the spectrum of $-i d \Pi(x)$. Q.E.D. 
We are now ready to implement Proposition 2 by determining a large class of type $\mathrm{F}$ groups.

If $K$ is a compact semisimple Lie group, and $T$ is a maximal torus of $K$, then the Weyl group $W(K)$ is $N(T) / T$, where $N(T)$ is the normalizer of $T$. We say $-1 \in W(K)$ if the automorphism $t \rightarrow t^{-1}$ of $T$ is in $W(K)$.

PROPOSITION 3. The following groups are type $\mathrm{F}$ :

(i) All complex semisimple Lie groups.

(ii) Any real semisimple Lie group $G$ for which $-1 \in W(K)$.

Proof. Part (ii) is an unpublished result due to B. Kostant. As to the proof of part (i), it is easy to verify that $S L_{2}(C)$ is of type F. Now let $G$ be any complex semisimple Lie group and let $x$ be an ad-nilpotent element of $g_{0}$. By the Jacobson-Morosov Lemma [3] there exists a three-dimensional noncompact subalgebra $t_{0}$ of $g_{0}$ which contains $x$. But $t_{0}$ is the Lie algebra of $S L_{2}(C)$ and the result follows immediately from the corresponding fact for $S L_{2}(C)$. Q.E.D.

It is now a simple matter to check when $-1 \in W(K)$ for a real semisimple Lie group. An examination of the list of all such groups $G$, [2], reveals that $-1 \in W(K)$ in all but the following cases: $S L_{n}(R)$ for $n \equiv 2$ $(\bmod 4), S O(p, q)$ for $p, q \equiv 2(\bmod 4), E_{6(2)}, E_{7(7)}$, and any group of automorphisms of a bounded symmetric domain. Thus by Propositions $1,2,3$ we conclude that Theorem 1 is valid for all complex semisimple Lie groups, and all but the above mentioned real semisimple Lie groups.

3. In this section we prove Theorem 1 for those groups not covered in $\S 2$.

Proposition 4. Let $G$, II be as in Theorem 1. If $x \in g_{0}$ can be embedded in a three-dimensional nilpotent algebra, with generators $x, y$ and relations $[x, y]=z,[x, z]=[y, z]=0$, then the spectrum of $-i d \Pi(x)$ is two-sided.

Proof. Il restricted to the nilpotent subgroup generated by $x, y, z$ decomposes as a direct integral of irreducible representations $\mathrm{II}_{\lambda}$, where $d \Pi_{\lambda}(z)=\lambda I$. These representations fall into two classes [4]. First is a series of one-dimensional representations when $\lambda=0$. Second are infinitedimensional representations for $\lambda \neq 0$ in which $-i d \Pi_{\lambda}(x)$ acts as the differentiation operator on a space of square integrable Hilbert space valued functions on $(-\infty, \infty)$. Thus for each $\lambda \neq 0,-i d \Pi_{\lambda}(x)$ has twosided spectrum, and clearly the direct integral of such representations has the same property. Finally, notice that the representations corresponding to $\lambda=0$ cannot occur. For if $d \Pi_{\lambda}(z)=0,\left.\Pi\right|_{\exp (t z)}$ contains the trivial onedimensional representation of $(\exp (t z))$. But a lemma of Jacobson [3] implies that $z$ is ad-nilpotent. Thus $(\exp (t z))$ is noncompact, and by [5], 
$\Pi$ must then contain a $G$-invariant vector. But $\Pi$ is irreducible, so this cannot occur. Q.E.D.

In order to apply Proposition 4, we prove

Proposition 5. Let $G$ be a real simple Lie group with a split real Lie algebra $g_{0}$ of rank greater than one. Assume the Dynken diagram of $g_{0}$ has no double bonds. Then any ad-nilpotent element $x \in g_{0}$, which is not the root vector corresponding to the top root, can be embedded in a three-dimensional nilpotent subalgebra $\{x, y, z\}$ where $[x, y]=z,[x, z]=[y, z]=0$.

Proof. Any nilpotent element of $g_{0}$ is conjugate by an inner automorphism of $G$ to an element of $n_{0}$, the nilpotent component of $g_{0}$ in the Iwasawa decomposition. Thus we may and will assume that $x \in n_{0}$, and hence $x=\sum_{\alpha} e_{\alpha}$, where $\alpha$ ranges over the set of positive roots in some given order. Recall that the level of a root $\alpha=\sum k_{i} \alpha_{i}, \alpha_{i}$ simple roots, is $\sum\left|k_{i}\right|$. Given $x=\sum c_{\alpha} e_{\alpha}$, choose a root of $\beta$ of highest level such that $\left[x, e_{\beta}\right] \neq 0$. Clearly such roots exist since $x$ is not the top root. Then $\left[x, e_{\gamma}\right]=0$ if level $(\gamma)>\operatorname{level}(\beta)$. We assert $x, e_{\beta},\left[x, e_{\beta}\right]$ is the three-dimensional nilpotent subalgebra.

For the rest of this proof, all sums will be over positive roots $\alpha$ such that $\alpha+\beta$ is a root. Now $\left[x, e_{\beta}\right]=\sum d_{\alpha+\beta} e_{\alpha+\beta}$, so

$$
\left[\left[x, e_{\beta}\right], e_{\beta}\right]=\sum d_{\alpha+\beta}\left[e_{\alpha+\beta}, e_{\beta}\right] .
$$

However, $\left[e_{\alpha+\beta}, e_{\beta}\right]=0$ for all $\alpha>0$ since $\alpha+2 \beta$ is never a root in any algebra whose Dynken diagram has no double bonds. Thus $\left[\left[x, e_{\beta}\right], e_{\beta}\right]=0$. Next $\left[\left[x, e_{\beta}\right], x\right]=\left[\sum d_{\alpha+\beta} e_{\alpha+\beta}, x\right]=\sum d_{\alpha+\beta}\left[e_{\alpha+\beta}, x\right]$. But level $(\alpha+\beta)>$ level $(\beta)$, hence $\left[e_{\alpha+\beta}, x\right]=0$ for all $\alpha>0$. Thus $\left[\left[x, e_{\beta}\right], x\right]=0$. Q.E.D.

Proposition 6. Theorem 1 is valid for $S L_{n}(R)$, all $n>2, E_{6(2)}$ and $E_{7(7)}$.

Proof. Observe first that Proposition 5 applies directly to $S L_{n}(R)$, $n>2$ and $E_{7(7)}$. On the other hand, while the Lie algebra $e_{6(2)}$ of $E_{6(2)}$ is the quasi-split real form, its nilpotent Iwasawa component is closely related to that of the split real form, and a slight modification of the above argument shows Proposition 5 valid in this case also. Thus by Propositions 4 and 5, any ad-nilpotent element of any of the above algebras which is not $c_{\gamma} e_{\gamma}$, where $\gamma$ is the top root, will have two-sided spectrum in any representation $d \Pi$.

Suppose $x=c_{\gamma} e_{\gamma}$. Since the corresponding Lie algebras are simple, there exists an element $w$ of the Weyl group mapping $\gamma$ onto a simple root. Now $w$ defines an inner automorphism of $g_{0}$ which conjugates $e_{\gamma}$ to a root vector corresponding to a simple root. But clearly any element conjugate to an element with two-sided spectrum has two-sided spectrum. Q.E.D. 
Finally, we complete the proof of Theorem 1 with the following proposition.

Proposition 7. Theorem 1 is valid for all groups $S O(n, m), m \leqq n$, $n, m \neq 2$.

Proof. If $g_{0}=k_{0}+h_{0}+n_{0}$ is the Iwasawa decomposition of $g_{0}$ [2], then any ad-nilpotent element of $g_{0}$ is conjugate by $\mathrm{Ad} G$ to an element of $n_{0}$. Thus it suffices to consider elements $n_{0}$.

The proof requires two cases.

Case 1. $m, n>2$. It is readily verified in this case that each nilpotent element of a basis for $n_{0}$ can be flipped by a suitable diagonal element of $S O(n, m)^{0}$, moreover the conjugating automorphisms may be chosen so as to either flip or leave invariant the other basis elements. Writing nilpotent elements as linear combinations of basis elements, we can show by induction on the number of nonzero coefficients that any nilpotent element has two-sided spectrum.

Case 2. $S O(n, 1), n \geqq 3$. Here we can show that every nilpotent element can be flipped. Q.E.D.

The above procedure breaks down for $S O(2,1)$, for there is no nontrivial element of $S O(1)$. Also observe that $S O(n, 1)$ is of type $\mathrm{F}$ for all $n \geqq 3$, even when $n \equiv 2(\bmod 4)$. Thus we have type $\mathrm{F}$ groups for which $-1 \notin$ Weyl group of the maximal compact subgroup. Q.E.D.

Finally observe that Theorem 1 may be extended from simple to semisimple Lie groups, and from irreducible to arbitrary unitary representations. The proof of the following is immediate.

THEOREM 2. Let $G$ be a connected semisimple Lie group such that no simple factor of $G^{*}$ is a group of automorphisms of a bounded symmetric domain. Let $I I$ be a continuous unitary representation of $G$ with no $G$ invariant vectors. Then if $x \in g_{0}$ generates a totally noncompact oneparameter subgroup $(\exp (t x)),-i d \Pi(x)$ has two-sided spectrum.

4. In this section we shall prove the following theorem.

THEOREM 3. Let $G$ be a connected simple Lie group of automorphisms of a bounded symmetric domain. There exists an $x \in g_{0}$ such that $-i d \mathrm{II}(x)$ has one-sided spectrum for any representation II of the holomorphic discrete series.

We begin with some algebraic preliminaries. Let $G$ be a simple Lie group of a bounded symmetric domain, with Lie algebra $g_{0}$. Let $g_{0}=$ $k_{0}+h_{0}+n_{0}$ be its Iwasawa decomposition, and let $g, k, n$ be the complexifications of $g_{0}, k_{y}, n_{0}$. Then $n=\Sigma_{\alpha} n_{x}$ is the root space decomposition of $n$, 
where $\alpha$ ranges over a set of positive roots of a Cartan subalgebra $h \supset h_{0}$ which do not vanish on $h_{0}$. It is known [8] that the top root $\gamma$ of $h$ does not vanish on $h_{0}$, and so $\left(e_{\gamma}\right) \subseteq n$. Clearly, we also have $\left(e_{\gamma}\right) \subseteq z(n)$, where $z(n)$ denotes the center of $n$.

Let $\sigma$ denote the conjugate linear involution of $g$ fixing $g_{0}$. A simple computation shows that $e_{\gamma}$ and $\sigma\left(e_{\gamma}\right)$ are both root vectors of $\gamma$, and since the root spaces of $h$ are one dimensional, we must have $\sigma\left(e_{\gamma}\right)=c e_{\gamma}$, for some constant $c$. But as $\sigma$ is an involution, its eigenvalues are \pm 1 . Thus either $\sigma\left(e_{\gamma}\right)=e_{\gamma}$, and $e_{\gamma} \in g_{0}$, or $\sigma\left(i e_{\gamma}\right)=i e_{\gamma}$, and $i e_{\gamma} \in g_{0}$. Thus either $e_{\gamma}$ or $i e_{\gamma}$ is contained in $z\left(n_{0}\right)=z(n) \cap g_{0}$.

Now let $z$ be the element of $\left\{e_{\gamma}, i e_{\gamma}\right\}$ contained in $z\left(n_{0}\right)$. Let $A=$ $\exp h_{0}$. If $a \in A$, it is immediate that $\operatorname{Ad}(a) z=e^{\gamma(x)} z, a=\exp (x)$, so that $\{\operatorname{Ad}(a) z \mid a \in A\}=\{t \cdot z \mid$ all $t>0\}$.

Proof of TheOrem 2. Let $U$ be any representation of the holomorphic discrete series for $G$, as constructed by Harish-Chandra [1]. Let $N$ be the nilpotent subgroup of $G$ with Lie algebra $n_{0}$, so that $A N$ is a Borel subgroup. Vergne and Rossi [7] have shown that $\left.U\right|_{A N}$ is a multiple of an irreducible representation $\Pi$ of $A N$. Consider now $\left.\Pi\right|_{N}$. Since $N$ is normal in $A N,\left.\Pi\right|_{N}$ decomposes as a direct integral of irreducible representations of $N ;\left.\Pi\right|_{N}=\int_{0} \Pi_{\Lambda} d \Lambda$ where 0 is an orbit of $A$ in the dual of $N$ [4]. Observe that $d \Pi_{\Lambda}$ is scalar on $(z)$.

Finally consider $\Pi$ restricted to $(\exp (t z))$. We have $\left.\Pi\right|_{\exp (t z)}=\int_{\Omega} \Pi_{\lambda} d \lambda$, where $\Pi_{\lambda}(\exp (t z))=e^{i \lambda(z)}, \lambda$ a linear functional on $(z)$, and $\Omega$ an orbit of $(z)^{*}$, the dual of $(z)$, under the dual action of $A$. Here of course $\Pi_{\lambda}$ is just the restriction of $\Pi_{\Lambda}$ to $(\exp (t z))$.

Now let $\rho$ denote the dual representation of $A$ on $(z)^{*}$. If $\lambda_{0} \in \Omega$, $a=\exp (x) \in A$, we have $\rho(a) \lambda_{0}(z)=\lambda_{0}\left(\operatorname{ad}\left(a^{-1}\right) z\right)=\lambda_{0}\left(e^{-\gamma(x)} z\right)=e^{-\gamma(x)} \lambda_{0}(z)$, from which it is clear that $\Omega=\left\{c \lambda_{0}: c>0\right\}$. Hence the spectrum of $-i d \Pi(z)$ is $\left\{c \lambda_{0}(z): c>0\right\}$ and is thus either $(-\infty, 0)$ or $(0, \infty)$. This completes the proof of Theorem 3 .

\section{REFERENCES}

1. Harish-Chandra, Representations of semi-simple Lie groups. V, Amer. J. Math. 78 (1956), 1-41. MR 18, 490.

2. S. Helgason, Differential geometry and symmetric spaces, Pure and Appl. Math., vol. 12, Academic Press, New York, 1962. MR 26 \#2986.

3. N. Jacobson, Lie algebras, Interscience Tracts in Pure and Appl. Math., no. 10, Interscience, New York, 1962. MR 26 \#1345.

4. A. A. Kirillov, Unitary representations of nilpotent Lie groups, Uspehi Mat. Nauk 17 (1962), no. 4 (106), 57-110=Russian Math. Surveys 17 (1962), no. 4, 53-104. MR 25 \#5396.

5. C. C. Moore, Ergodicity of flows on homogeneous spaces, Amer. J. Math. 88 (1966), 154-178. MR 33 \#1409. 
6. E. Nelson, Analytic vectors, Ann. of Math. (2) 70 (1959), 572-615. MR 21 \#5901.

7. H. Rossi and M. Vergne, Functions holomorphes de carré sommable sur un domaine de Siegel et étude de la série discrete holomorphe, C. R. Acad. Sci. Paris 275 (3 juillet 1972).

8. W. Schmid, Die Randwerte holdmorpher Funktionen auf hermitesch symmetrischen Raümen, Invent. Math. 9 (1969/70), 61-80. MR 41 \#3806.

Atkinson College, York University, Downsview, Ontario, Canada 